\title{
High-intensity resistance exercise is not as effective as traditional high-intensity interval exercise for increasing the cardiorespiratory response and energy expenditure in recreationally active subjects
}

\author{
Laura Järvinen $^{1} \cdot$ Sofi Lundin Petersdotter ${ }^{1} \cdot$ Thomas Chaillou $^{1} \mathbb{D}$ \\ Received: 21 June 2021 / Accepted: 12 November 2021 / Published online: 19 November 2021 \\ (c) The Author(s) 2021
}

\begin{abstract}
Purpose Traditional high-intensity interval exercise (HIIE) highly stimulates the cardiorespiratory system and increases energy expenditure (EE) during exercise. High-intensity resistance exercise (HIRE) has become more popular in recreationally active subjects. The physiological responses to HIRE performed with light or moderate load is currently largely unknown. Here, we examined the effect of the type of interval exercise [HIRE at 40\% (HIRE40) and 60\% (HIRE60) 1-RM vs. traditional HIIE] on the cardiorespiratory response and EE during and after exercise.

Methods Fifteen recreationally active adults randomly completed traditional HIIE on an ergocyle, HIRE40 and HIRE60. The sessions consisted of two sets of ten 30-s intervals (power at $100 \% \mathrm{VO}_{2 \max }$ during HIIE; maximal number of repetitions for 10 different free-weight exercises during HIRE40 and HIRE60) separated by 30-s active recovery periods. Gas exchange, heart rate (HR) and $\mathrm{EE}$ were assessed during and after exercise.

Results $V \mathrm{O}_{2 \text { mean }}, V \mathrm{O}_{2 \text { peak }}, \mathrm{HR}_{\text {mean }}$, the time spent above $90 \% V_{2 \max }$ and $\mathrm{HR}_{\text {max }}$, and aerobic EE were lower in both HIRE sessions compared with HIIE $(P<0.05)$. Anaerobic glycolytic contribution to total exercise EE was higher in HIRE40 and HIRE60 compared with HIIE $(P<0.001)$. EE from excess post-exercise oxygen consumption (EPOC) was similar after the three sessions. Overall, similar cardiorespiratory responses and EE were found in HIRE40 and HIRE60.

Conclusions HIRE is not as effective as HIIE for increasing the cardiorespiratory response and EE during exercise, while EPOC remains similar in HIRE and HIIE. These parameters are not substantially different between HIRE40 and HIRE60.
\end{abstract}

Keywords Maximal oxygen uptake $\cdot$ Aerobic fitness $\cdot$ Resistance exercise $\cdot$ Heart rate $\cdot$ RPE $\cdot$ HIIT

$\begin{array}{ll}\text { Abbreviations } \\ \text { 1-RM } & \text { 1-Repetition maximum } \\ \text { ATP } & \text { Adenosine triphosphate } \\ \text { EE } & \text { Energy expenditure } \\ \text { EPOC } & \text { Excess post-exercise oxygen consumption } \\ \text { HIIE } & \text { High-intensity interval exercise } \\ \text { HIRE } & \text { High-intensity resistance exercise } \\ \text { HIRE40 } & \text { High-intensity resistance exercise at 40\% } \\ & \text { 1-repetition maximum }\end{array}$

Communicated by William J. Kraemer.

Laura Järvinen and Sofi Lundin Petersdotter have equally contributed and are co-first authors.

Thomas Chaillou

thomas.chaillou@oru.se

1 School of Health Sciences, Örebro University, 70182 Örebro, Sweden
HIRE60 High-intensity resistance exercise at $60 \%$ 1-repetition maximum

HR Heart rate

$\mathrm{HR}_{\max } \quad$ Maximal heart rate

$\mathrm{HR}_{\text {mean }} \quad$ Mean heart rate

$\mathrm{HR}_{\text {peak }} \quad$ Peak heart rate

PC Phosphocreatine

Pmax Power eliciting maximal oxygen uptake

RM ANOVA One-way repeated measures analysis of variance

RPE Rate of perceived exertion

RER Respiratory exchange ratio

$\mathrm{RER}_{\text {max }} \quad$ Maximal respiratory exchange ratio

$\mathrm{RER}_{\text {mean }} \quad$ Mean respiratory exchange ratio

$\mathrm{RER}_{\text {peak }} \quad$ Peak respiratory exchange ratio

T90\% $\mathrm{HR}_{\max }$ Time spent above $90 \%$ maximal heart rate

$\mathrm{T} 90 \% \mathrm{VO}_{2 \max } \quad$ Time spent above $90 \%$ maximal oxygen uptake

VE Ventilation 


$\begin{array}{ll}V E_{\text {max }} & \text { Maximal ventilation } \\ \mathrm{VE}_{\text {peak }} & \text { Peak ventilation } \\ V \mathrm{O}_{2} & \text { Oxygen uptake } \\ V \mathrm{O}_{2 \text { max }} & \text { Maximal oxygen uptake } \\ V \mathrm{O}_{2 \text { mean }} & \text { Mean oxygen uptake } \\ V \mathrm{O}_{2 \text { peak }} & \text { Peak oxygen uptake } \\ \eta^{2} & \text { Eta squared } \\ {\left[\mathrm{La}^{-}\right]} & \text {Blood lactate concentration }\end{array}$

\section{Introduction}

Endurance exercise promotes the stimulation of the cardiorespiratory system and increases energy expenditure (EE) (Pinckard et al. 2019). In addition, EE remains elevated over the early period following endurance exercise due to high metabolic demands, which can be evaluated by the excess post-exercise oxygen consumption (EPOC) (Gaesser and Brooks 1984). Endurance training is highly recommended for improving cardiovascular fitness and improving body weight control (Skrypnik et al. 2015; Pinckard et al. 2019). Moderate-intensity continuous exercise and high-intensity interval exercise (HIIE) are the main forms of endurance exercise. HIIE appears to be equally, if not more effective, for improving maximum oxygen uptake $\left(V \mathrm{O}_{2 \max }\right.$, a physiological determinant of cardiovascular fitness) as prolonged moderate-intensity continuous exercise, despite a limited training volume and time commitment (Gibala 2020). HIIE can also increase EPOC more importantly than isocaloric continuous exercise (Jung et al. 2019).

Traditional HIIE, commonly performed in individual endurance sports (e.g., running, cycling, rowing, etc.), is characterized by high-intensity intervals (i.e., performed near the power/speed eliciting $V \mathrm{O}_{2 \max }$ ), interspaced with periods of recovery. Several variables define a HIIE session, such as the intensity and duration of the working/recovery periods, and the number of sets (Buchheit and Laursen 2013). Over the last 20 years, numerous studies investigated the cardiorespiratory changes during HIIE sessions consisting of short working intervals (i.e., $30 \mathrm{~s}$ ) performed at intensities near $V \mathrm{O}_{2 \max }$ (Billat et al. 2000; Thevenet et al. 2007a, b, 2008; Millet et al. 2003b; Rozenek et al. 2007; Rønnestad and Hansen 2016). These studies indicate that HIIE with short intervals are highly effective in eliciting prolonged times spent at a high percentage of $V \mathrm{O}_{2 \max }$ (i.e. $\geq 90 \%$ $V \mathrm{O}_{2 \max }: \mathrm{T} 90 \% V \mathrm{O}_{2 \max }$ ) or at a high percentage of maximal heart rate (HR) (i.e. $\geq 90 \% \mathrm{HR}_{\max }$ : $\mathrm{T} 90 \% \mathrm{HR}_{\max }$ ). HIIE with longer intervals could also be relevant for sustaining elevated $\mathrm{T} 90 \% \mathrm{VO}_{2 \max }$ and $\mathrm{T} 90 \% \mathrm{HRmax}$, but whether it is more effective than HIIE with short intervals remains controversial (Rønnestad and Hansen 2016; Millet et al. 2003a). It has been proposed that the total time spent at high intensity is an important criterion to assess the effectiveness of a training program to improve $V \mathrm{O}_{2 \max }$ (Thevenet et al. 2007a; Midgley et al. 2006; Buchheit and Laursen 2013), and training interventions with traditional HIIE are very effective and time-efficient for developing $\mathrm{VO}_{2 \max }$ in healthy populations (Midgley et al. 2006; Wen et al. 2019; Gibala 2020). In addition, this training method highly increases EE during and after exercise (Skelly et al. 2014), and could have beneficial effects on body weight and body composition (Keating et al. 2017).

Recently, high-intensity resistance exercise (HIRE) and other types of high-intensity functional exercise have become more popular in recreationally active individuals (Claudino et al. 2018; Feito et al. 2018). HIRE consists of resistance exercises that combine numerous multiple-joint exercises performed at high intensity (usually defined as the maximal number of repetitions during a fixed period) with a load and in a circuit-type manner. High-intensity functional exercises are very attractive in recreationally active subjects because they can be modified to any fitness level, elicit varied muscle recruitment, and could be beneficial for improving muscle strength and muscle endurance (Munoz-Martinez et al. 2017; McRae et al. 2012; Myers et al. 2015; Feito et al. 2018). This training method also appears effective for developing $V \mathrm{O}_{2 \max }$ (Munoz-Martinez et al. 2017), but it is uncertain whether HIRE that recruit multiple and large muscle groups can elicit prolonged $\mathrm{T} 90 \% \mathrm{VO}_{2 \max }$ and $\mathrm{T} 90 \% \mathrm{HR}_{\max }$, thereby imposing an optimal stimulus for improving $V \mathrm{O}_{2 \max }$ (Thevenet et al. 2007a; Midgley et al. 2006; Buchheit and Laursen 2013). Although the stimulation of the cardiorespiratory system may be lower during HIRE (i.e., combination of several resistance exercise bouts) compared with traditional HIIE (i.e., repetitions of intense aerobic exercises with a cyclic movement pattern), resulting in a lower EE from the aerobic system, these exercises could be more anaerobically demanding (Benito et al. 2016). It is, therefore, important to also consider the contribution of the anaerobic system to the total EE during exercise.

HIRE is generally practiced with loads between 20 and 80\% 1-repetition maximum (1-RM) (Munoz-Martinez et al. 2017), and intervention studies showing an improvement of $V \mathrm{O}_{2 \max }$ usually included HIRE sessions performed with loads between 30 and 65\% 1-RM (Munoz-Martinez et al. 2017). Due to the negative relationship between the number of repetitions and the \% 1-RM in free-weight exercises (Shimano et al. 2006), it is evident that recreationally active subjects should perform HIRE with submaximal loads to accomplish a high number of repetitions and total working load, which may therefore highly solicit the cardiorespiratory system. To date, it is unclear whether HIRE executed at maximal effort (i.e., maximal number of repetitions during a fixed period) should be performed with light load (i.e., $40 \% 1-\mathrm{RM})$ or moderate load (60\% 1-RM) to maximize $\mathrm{T} 90 \% \mathrm{VO}_{2 \max }$ and $\mathrm{T} 90 \% \mathrm{HR}_{\max }$. Furthermore, it remains to 
be elucidated whether HIRE with light load and moderate load differently affect aerobic/anaerobic glycolytic exercise $\mathrm{EE}$ and EPOC.

Therefore, the aim of this study was to examine the effect of the type of interval exercise (HIRE with light load or moderate load vs. traditional HIIE) on the cardiorespiratory response (such as $V \mathrm{O}_{2}, \mathrm{~T} 90 \% V \mathrm{O}_{2 \max }, \mathrm{HR}, \mathrm{T} 90 \% \mathrm{HR}_{\max }$ ) and EE during and after exercise in young recreationally active participants. We hypothesized that the cardiorespiratory response and aerobic exercise EE would be lower during the HIRE sessions than the HIIE session. In addition, we hypothesized that the anaerobic glycolysis exercise EE and EPOC would be greater during HIRE sessions than during HIIE session. Finally, these physiological parameters may be higher during HIRE with moderate load than HIRE with light load.

\section{Materials and methods}

\section{Participants}

Twenty-one recreationally active participants were initially recruited from two local fitness centers (Örebro, Sweden). The inclusion criteria were: aged between 20 and 40 years, being healthy and free of diagnosed cardiovascular and metabolic diseases, performing structured exercise training at least twice a week (with a total of light/moderate-intensity physical activity $>150$ min per week), and having at least 1-year experience in resistance training. The exclusion criteria were: any disease, injury or another condition that could compromise the ability to perform the physical tests. Due to the withdrawal of five subjects and the exclusion of another one, 15 subjects $(25.5 \pm 3.3$ years; 10 males and 5 females) were included in the study. The height, body mass, percentage of body fat (as described below), body mass index were $178.0 \pm 10.8 \mathrm{~cm}, 81.4 \pm 10.5 \mathrm{~kg}, 21.3 \pm 7.4 \%$ and $25.8 \pm 3.4 \mathrm{~kg} / \mathrm{m}^{2}$, respectively. The participants were fully informed of the study procedures and a written informed consent was obtained from all participants. This study was performed in accordance with the declaration of Helsinki and was approved by the regional ethics committee of Uppsala (DNR 2018/321).

\section{Experimental design}

The current study employed a randomized crossover design. The experiments consisted of seven visits to the laboratory or local fitness centers. All exercise sessions were separated by at least two days and the subjects did not perform any strenuous exercise at least $48 \mathrm{~h}$ prior to each exercise session. During the first visit, participants were familiarized with the equipment and anthropometric measurements and resting $\mathrm{VO}_{2}$ were determined. In visits two to three, 1-repetition maximum (1-RM) was determined for the ten exercises executed during the HIRE sessions (see description below). During the fourth visit, a maximal incremental cycling test was performed to determine $V \mathrm{O}_{2 \max }$ and the power eliciting $V \mathrm{O}_{2 \max }\left(P_{\max }\right)$. In visits five to seven, the participants performed three HIIE sessions in a random order. Each participant performed the physical tests and exercise sessions at a similar time of the day and was instructed to follow a similar diet before each test.

\section{Familiarization session}

The familiarization session was performed in the morning after at least 12-h fasting. Body height was measured using standard procedures, and body mass and percentage of body fat were determined using bioelectrical impedance analysis (MC-780MA, Tanita Corporation, Japan). It is noteworthy that during the familiarization session, participants were only familiarized with the equipment used, but not with the physical tests and exercise sessions performed afterwards. For the determination of resting $V \mathrm{O}_{2}$, participants lied in a supine position for $15 \mathrm{~min}$. $V \mathrm{O}_{2}$ was assessed during this period with a gas analyzer (Jaeger Oxycon Mobile, Vyaire Medical, USA), and resting $V \mathrm{O}_{2}$ calculated from the values obtained during the last $5 \mathrm{~min}$ was $3.35 \pm 0.29 \mathrm{~mL} / \mathrm{kg} / \mathrm{min}$.

\section{Selection of the weight exercise movements and determination of 1-RM}

Ten exercises were selected for the HIRE sessions (Table 1), consisting of compound exercises that recruit multiple and large muscle groups. These exercises were executed with either a loaded barbell or dumbbells. Most of these exercises activated muscle groups from the lower limbs, trunk and upper limbs, as described in Table 1. The specific order of execution was chosen to limit the recruitment of similar muscle groups during successive exercises and to limit the accumulation of excessive fatigue that would prevent the proper execution of the movements. Since gas exchanges were measured during the entire HIRE sessions, it was important to select exercises that could be properly executed with the mobile gas analyzer. The participants wore the vest containing the device during all exercises, except during the last two exercises (i.e., chest press and hip thrust) due to the specific position.

1-RM for the 10 exercises of the HIRE sessions was estimated during tests performed at submaximal load (visits 2 and 3; 5 exercises per visit). Briefly, after a proper warm-up, subjects performed the tests with a submaximal load until failure (load estimated to be lifted 2-10 times). These tests (as well as the HIRE sessions) were performed at the local fitness center where participants had their membership. The 
Table 1 Weight exercise movements

\begin{tabular}{|c|c|c|c|}
\hline Order & Movement & Description & Activation \\
\hline 1 & Deadlift & Traditional BB deadlift from the floor & $\operatorname{LL}(+++), \mathrm{T}(++), \mathrm{UL}(++)$ \\
\hline 2 & Step up & DB Step up to a bench & $\mathrm{LL}(+++), \mathrm{T}(+), \mathrm{UL}(++)$ \\
\hline 3 & Thruster & Front squat followed immediately by push press with DB & $\mathrm{LL}(+++), \mathrm{T}(++), \mathrm{UL}(+++)$ \\
\hline 4 & Pendlay row & Pronated grip BB row from the floor & $\operatorname{LL}(+), \mathrm{T}(+++), \mathrm{UL}(+++)$ \\
\hline 5 & Reverse fly & Bent-over DB fly with slightly bent elbows & $\mathrm{LL}(+), \mathrm{T}(+++), \mathrm{UL}(+++)$ \\
\hline 6 & Romanian deadlift & Straight leg BB deadlift from hip to under knee position & $\mathrm{LL}(+++), \mathrm{T}(++), \mathrm{UL}(++)$ \\
\hline 7 & Bent-over row & Supinated grip BB row from above the knee & $\mathrm{LL}(+), \mathrm{T}(+++), \mathrm{UL}(+++)$ \\
\hline 8 & Lunges & Forward lunge with DB & $\mathrm{LL}(+++), \mathrm{T}(++), \mathrm{UL}(++)$ \\
\hline 9 & Chest press & Flat bench chest press with DB and feet up on the bench & $\mathrm{LL}(/), \mathrm{T}(++), \mathrm{UL}(+++)$ \\
\hline 10 & Hip thrust & Thrust movement with BB placed on anterior pelvis & $\mathrm{LL}(+++), \mathrm{T}(+++), \mathrm{UL}(/)$ \\
\hline
\end{tabular}

Movements performed with a loaded barbell (BB) or with dumbbells (DB). Each exercise was performed during $30 \mathrm{~s}$ in both sets 1 and set 2 , in the order presented in the left column

$L L$ muscles of the lower limbs, $T$ trunk muscles, $U L$ muscles of the upper limbs, + light activation, ++ moderate activation, +++ high activation, / no or minor activation

Brzycki equation was used to estimate 1-RM (Mayhew et al. 1995). The load used during the HIRE sessions was calculated for each exercise according to 1-RM estimation (40\% 1-RM during HIRE40 and 60\% 1-RM during HIRE60).

\section{Maximal incremental cycling test}

$\mathrm{VO}_{2 \max }$ was determined during a maximal incremental cycling test performed on a cycling ergometer (Monark LC6, Monark Exercise AB, Sweden). After a 5-min warm-up, power was increased by $20 \mathrm{~W}$ every minute until exhaustion. The subjects maintained a pedaling cadence above $70 \mathrm{rpm}$ through the entire test. Expired gases were analyzed using a gas analyzer (Jaeger Oxycon Mobile, Vyaire Medical, USA) connected with JLAB software, and HR was recorded simultaneously using a coded transmitter belt (T31, Polar, Finland). Lactate concentration from fingertip blood sample ([La $\left.{ }^{-}\right]$) was measured with a portable device (Lactate Plus, Cardioworld, Germany) three minutes after the completion of the test. The rate of perceived exertion (RPE, 6-20 Borg's scale) was determined after each stage and at the end of the test.

Participants were considered to have reached their $V \mathrm{O}_{2 \max }$ when at least three of the five following criteria were met: (1) a plateau of $V \mathrm{O}_{2}$ (i.e., change $\leq 2,1 \mathrm{~mL} / \mathrm{kg} / \mathrm{min}$ ), (2) a final respiratory exchange ratio (RER) $\geq 1.1$, (3) maximum heart rate within $10 \mathrm{bpm}$ of the age-predicted maximum [210-(0,65 ×age)], (4) a post-test $\left[\mathrm{La}^{-}\right] \geq 8.0 \mathrm{mmol} / \mathrm{L}$, and (5) $\mathrm{RPE} \geq 18 . V \mathrm{O}_{2 \max }$, maximal ventilation $\left(\mathrm{VE}_{\max }\right), \mathrm{HR}_{\max }$ and $\mathrm{RER}_{\max }$ were defined as the mean of the highest values obtained during six consecutive 5 -s periods (30 $\mathrm{s}$ in total) and are presented in Table 2. Power at $V \mathrm{O}_{2 \max }\left(P_{\max }\right)$ was the lowest load that allowed $V \mathrm{O}_{2 \max }$ to be reached. If $V \mathrm{O}_{2 \max }$ was reached within the first $30 \mathrm{~s}$ of a specific stage, $P_{\max }$ was calculated as the power at this stage minus $10 \mathrm{~W}$.

\section{Interval exercises}

The participants performed three exercise sessions in a random order and on three separate occasions. Each session

Table 2 Cardiorespiratory parameters during the maximal incremental cycling test and the three interval exercise sessions

\begin{tabular}{lccccc}
\hline & Incremental test & HIIE & HIRE40 & HIRE60 & $\eta^{2} / \underline{\text { Kendall W }}$ \\
\hline$V \mathrm{O}_{2 \text { max } / \text { peak }}(\mathrm{mL} / \mathrm{kg} / \mathrm{min})$ & $38.9 \pm 7.1$ & $41.8 \pm 6.6 \$$ & $33.7 \pm 5.2 \$ \$ * * *$ & $34.4 \pm 6.0 \$ * * *$ & 0.734 \\
$\mathrm{HR}_{\text {max/peak }}(\mathrm{bpm})$ & $188.5 \pm 10.1$ & $187.5 \pm 12.6$ & $180.3 \pm 14.6$ & $180.3 \pm 10.5 \$ *$ & $\underline{0.364}$ \\
$\mathrm{VE}_{\text {max } / \text { peak }}(\mathrm{L} / \mathrm{min})$ & $152.2 \pm 44.1$ & $132.0 \pm 20.3$ & $111.0 \pm 28.1 \$ \$ * *$ & $117.1 \pm 28.7 \$ \$ *$ & 0.598 \\
$\mathrm{RER}_{\text {max/peak }}$ & $1.31 \pm 0.08$ & $1.13 \pm 0.08 \$ \$ \$$ & $1.39 \pm 0.13 * *$ & $1.43 \pm 0.13 \$ *$ & 0.685 \\
\hline
\end{tabular}

Data are shown as mean $\pm \mathrm{SD}(N=11)$

$V O_{2}$ oxygen consumption, $H R$ heart rate, $V E$ ventilation, $R E R$ respiratory exchange ratio, $\eta^{2}$ eta squared, highlighted in italic in the right column. Kendall $\mathrm{W}$ underlined in the right column

$* P<0.05, * * P<0.01, * * * P<0.001$ : significantly different compared with HIIE. $\$ P<0.05, \$ \$ P<0.01, \$ \$ \$ P<0.001$ : significantly different compared with the incremental test 
began with a 10-min warm-up performed on a cycle ergometer at $60-70 \%$ maximum HR (determined during the maximal incremental test). The warm-up was followed by a 2-min transition period before the start of the session. Each exercise session consisted of two sets of ten 30-s working intervals interspaced with 30 -s recovery periods. The two sets were separated by 4-min active walking recovery (i.e., between the last working interval of set 1 and the first working interval of set 2) at a self-controlled pace. This 4-min resting period was included between the two sets to limit extensive fatigue and to allow the recreationally active participants to complete the entire session (i.e., 10 min work). The scheme of the exercise sessions (i.e., duration of the intervals and recovery periods, number of exercise bouts per set, and number of sets) was identical for the three sessions. After the completion of the last working interval in set 2, the subjects were instructed to sit and rest for $12 \mathrm{~min}$ to evaluate EPOC and EE derived from EPOC during the early recovery phase. $V \mathrm{O}_{2}$ did not reach resting $V \mathrm{O}_{2}$ (which was assessed in a lying position) after $12 \mathrm{~min}$. However, we decided to not extend the duration of the measurement to limit the discomfort after exercise and because $\mathrm{VO}_{2}$ was already stabilized at $\sim 5-6 \mathrm{~mL} / \mathrm{kg} / \mathrm{min}$ after $12 \mathrm{~min}$, a $V \mathrm{O}_{2}$ level used by others (Scott et al. 2011). Gas exchanges and HR were recorded during the warm-up and the exercise sessions, as described above. Blood lactate concentration was measured from fingertip at rest (before the warm-up) and three minutes after the two sets, and RPE (6-20 Borg's scale) was determined directly after the two sets. These two parameters were evaluated to obtain indications of exercise intensity and perceived effort (Zinoubi et al. 2018; Tiggemann et al. 2010; Rønnestad et al. 2020). In addition, RER and VE were assessed during both sets.

The HIIE session was performed on a cycle ergometer (Monark LC6, Monark Exercise AB). The intensity of the 30-s working intervals was set at $100 \% P_{\max }$ determined during the maximal incremental cycling test and the 30 -s recovery intervals were performed at $50 \% P_{\max }$. We selected this HIIE session because $30 \mathrm{~s} / 30 \mathrm{~s}$ interval exercises at these intensities are well-tolerated by endurance-trained subjects and are effective in eliciting prolonged $\mathrm{T} 90 \% \mathrm{VO}_{2 \max }$ and $\mathrm{T} 90 \% \mathrm{HR}_{\max }$ (Billat et al. 2000; Thevenet et al. 2007a, b, 2008; Millet et al. 2003b). As explained above, two sets of ten intervals interspaced with 4-min rest were used in our study (while $30 \mathrm{~s}$ intervals were performed until exhaustion by well-trained athletes in the later studies) to limit extensive fatigue and to allow our participants to achieve $10 \mathrm{~min}$ at high intensity. Indeed, results from an unpublished pilot study performed in our laboratory showed that recreationally active subjects $(N=7 ; 24.3 \pm 2.7$ years $)$ were able to complete this HIIE session and maintain high percentages of $V \mathrm{O}_{2 \max }$ and $\mathrm{HR}_{\max }$. Despite these preliminary tests, 4 of our 15 recreationally active participants were unable to complete the two sets of HIIE due to severe exhaustion. For this reason, these participants performed a second HIIE session (at least $48 \mathrm{~h}$ later) that consisted of two sets of ten 30-s working interval $\left(95 \% P_{\max }\right)$ interspaced with 30 -s recovery intervals $\left(50 \% P_{\max }\right)$.

The two HIRE sessions consisted of two sets of ten 30-s working intervals (intensity described below) separated by 30 -s recovery periods (time used to put down the loaded barbell or dumbbells, walk and be ready for the next exercise). Each exercise movement (described in Table 1) was performed during one 30-s working interval period in both sets. The order of the ten exercises is presented in Table 1 and the choice of the exercises was justified above. The exercises were executed at a load corresponding to $40 \%$ of 1 -RM during the HIRE40 session, and $60 \%$ of 1 -RM during the HIRE60 session. Our pilot study revealed that participants could perform on average 10-12 repetitions at $60 \%$ 1-RM during 30-s working intervals. Selecting a higher \% 1-RM would have limited the number of repetitions performed and thus would have certainly provoked important breaks during the working intervals. In opposite, selecting $40 \%$ 1-RM was a good option to increase the number of repetitions performed while maintaining a high total load lifted (see Table 5). The load used during the HIRE sessions was rounded to the closest available weight, which was never more than $1.2 \mathrm{~kg}$ below or above the calculated load. The subjects were familiarized with the exercises and the order of the exercises before starting the HIRE sessions. The participants were instructed to perform the 30-s working intervals of HIRE sessions at their maximal sustainable work intensity, aiming to execute the highest number of repetitions of each movement. The number of repetitions and the load lifted during each set was calculated to evaluate the cumulated work of HIRE40 and HIRE60 sessions.

Due to the specific nature of our exercise protocols, the performed work of the exercise sessions could not be matched for total work. Instead, our approach was based on the matching for the perceived effort (i.e. RPE) assessed directly after exercise, which applies more to real world training (Rønnestad et al. 2020). We validated this approach with our specific setting during a pilot experiment (as described above). This experiment showed that RPE was $19.0 \pm 0.8$ after a HIIE (same protocol as in the current study) and 19.4 \pm 1.4 after HIRE60 (same protocol as in the current study, except that five exercise movements were executed twice in each set) $(P=0.41)$, indicating that the perceived effort was similar in both sessions.

\section{Analysis of cardiorespiratory parameters, energy expenditure and EPOC during and after the exercise sessions}

The cardiorespiratory values were averaged over a 5-s period to determine $V \mathrm{O}_{2 \text { mean }}, V \mathrm{O}_{2 \text { peak }}, \mathrm{VE}_{\text {peak }}, \mathrm{HR}_{\text {mean }}, \mathrm{HR}_{\text {peak }}$, 


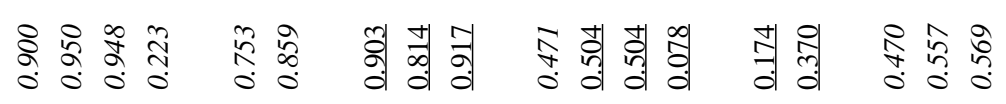

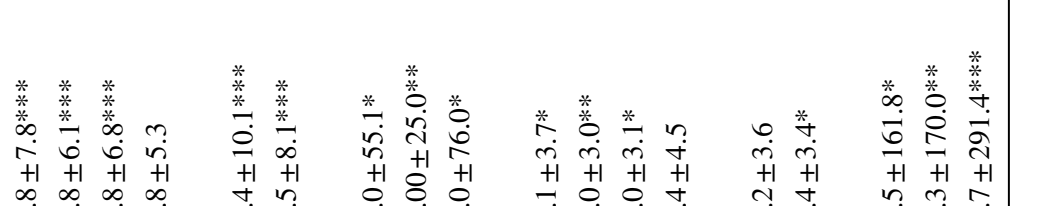

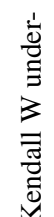

$\begin{array}{llll}\infty & \infty & \infty & \infty \\ \dot{b} & \dot{b} & \sim & \infty \\ 0 & \infty & 0\end{array}$

$\begin{array}{lll}+ & +1 \\ \infty & \ddots \\ \infty & \infty\end{array}$

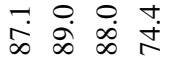

ปั

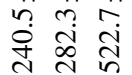

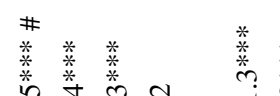

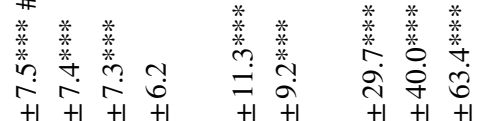

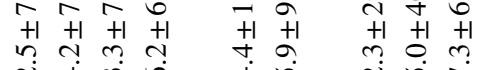

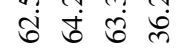

मे

तै थี

ค่

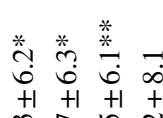

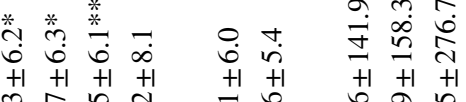

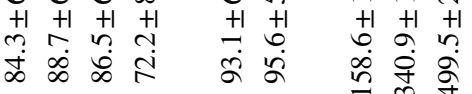

站

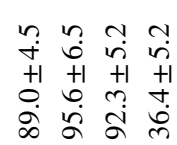

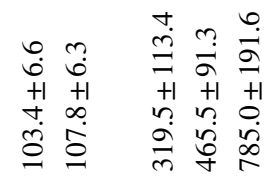

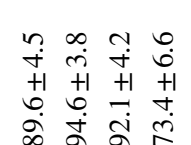

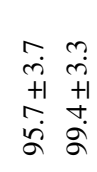

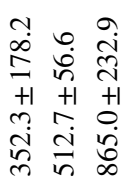

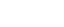

$$
\text { . }
$$$$
\text { (19) }
$$$$
\text { (1) }
$$

啇

8

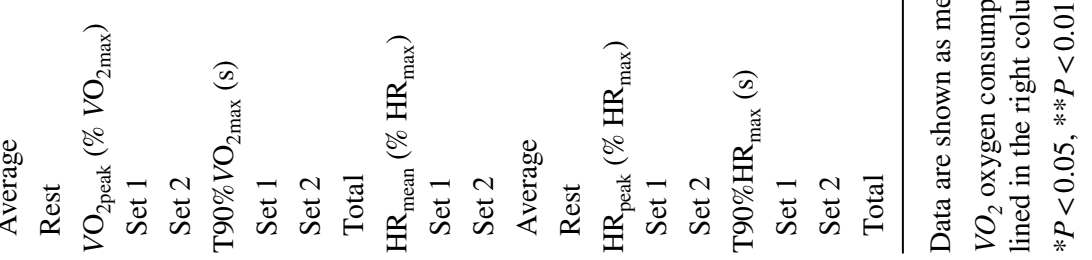


$\mathrm{RER}_{\text {mean }}$ and $\mathrm{RER}_{\text {peak }}$. The gas analyzer used in this study (Jaeger Oxycon Mobile, Vyaire Medical) is considered to be a reliable device, with coefficients of variation from 1.6 to $5.8 \%$ for $V \mathrm{O}_{2}$ and RER over a range of intensities (submaximal to maximal) (Rosdahl et al. 2010). $\mathrm{VO}_{2 \text { peak }}, \mathrm{HR}_{\text {peak }}$, $V E_{\text {peak }}$ and $R_{\text {peak }}$ were defined as the mean of the highest values obtained during six consecutive 5-s periods. $V_{2} \mathrm{O}_{2 \text { mean }}$ and $\mathrm{HR}_{\text {mean }}$ were calculated in both sets 1 and 2, during the entire interval exercise period (i.e. average of sets 1 and 2 , excluding the recovery period between the two sets). In Table $3, V O_{2 \text { peak }}$ and $V \mathrm{O}_{2 \text { mean }}$ were expressed as a percentage of $V \mathrm{O}_{2 \max }$ (determined during the maximal incremental test) and $\mathrm{HR}_{\text {peak }}$ and $\mathrm{HR}_{\text {mean }}$ were expressed as a percentage of $\mathrm{HR}_{\max }$ (determined during the maximal incremental test). $\mathrm{T} 90 \% V \mathrm{O}_{2 \max }$ and $\mathrm{T} 90 \% \mathrm{HR}_{\max }$ were calculated during each set and during the entire exercise session (i.e. cumulated time spent during sets 1 and 2).

The $\mathrm{VO}_{2}$ values were averaged over a 30 -s period to determine aerobic EE and EPOC. Three different caloric equivalents were used for the calculation of $\mathrm{EE}$. The caloric equivalent was set at $4.85 \mathrm{kcal} / \mathrm{L}$ of $\mathrm{O}_{2}$ during the warm-up, and at $5.05 \mathrm{kcal} / \mathrm{L}$ of $\mathrm{O}_{2}$ during the interval exercise period (sets 1 and 2) (Schaun et al. 2017). The caloric equivalent was set at $4.64 \mathrm{kcal} / \mathrm{L} \mathrm{O}_{2}$ during the between-set recovery period and the 12-min post-exercise period to exclude rapid glycolytic ATP resynthesis (Benito et al. 2016). Indeed, exercise anaerobic glycolytic EE was estimated using the $\mathrm{O}_{2}$ equivalent of blood lactate accumulation, as previously described (di Prampero and Ferretti 1999; Benito et al. 2016). During the three interval exercise sessions, $\Delta\left[\mathrm{La}^{-}\right]$ was calculated by subtracting resting values from peak $\left[\mathrm{La}^{-}\right]$ reached $3 \mathrm{~min}$ after exercise. $\Delta\left[\mathrm{La}^{-}\right]$was then converted to $\mathrm{O}_{2}$ equivalent values as $3 \mathrm{~mL} \mathrm{O} / \mathrm{kg}$ body mass $/ \mathrm{mmol}$ of $\Delta\left[\mathrm{La}^{-}\right]$(Benito et al. 2016). Exercise anaerobic glycolytic $\mathrm{EE}$ was calculated using the caloric equivalent of $5.05 \mathrm{kcal} / \mathrm{L}$ of $\mathrm{O}_{2}$. Total exercise EE was defined as the sum of aerobic $\mathrm{EE}$ and anaerobic glycolytic EE during sets 1 and 2. EPOC was calculated every min during the 12 -min post-exercise period using the following formula: $\mathrm{EPOC}(\mathrm{L} / \mathrm{min})=V \mathrm{O}_{2}$ post-exercise $(\mathrm{L} / \mathrm{min})$ - resting $\mathrm{VO}_{2}(\mathrm{~L} / \mathrm{min})$. EPOC was then converted to EE as $1 \mathrm{~L}$ of $\mathrm{O}_{2}=4.64 \mathrm{kcal}$ to exclude rapid glycolytic ATP resynthesis as part of the conversion of $\mathrm{O}_{2}$ uptake into EE (Benito et al. 2016). EPOC represents the $\mathrm{O}_{2}$ consumption for aerobic ATP turnover and an estimation of the fast resynthesis of ATP and phosphocreatine (PC) stores (Scott et al. 2011).

\section{Statistical analysis}

Data are presented as mean \pm standard deviation (SD). Statistical analyses were performed with Graphpad Prism (version 8.3) and SPSS Statistics (version 27; for analysis of effect size only). Shapiro-Wilk tests were used to check 
Fig. $1 \mathrm{VO}_{2}(\mathbf{A})$ and $\mathrm{HR}(\mathbf{B})$ during the three interval exercise sessions. Data are the mean values obtained from 11 participants.
A

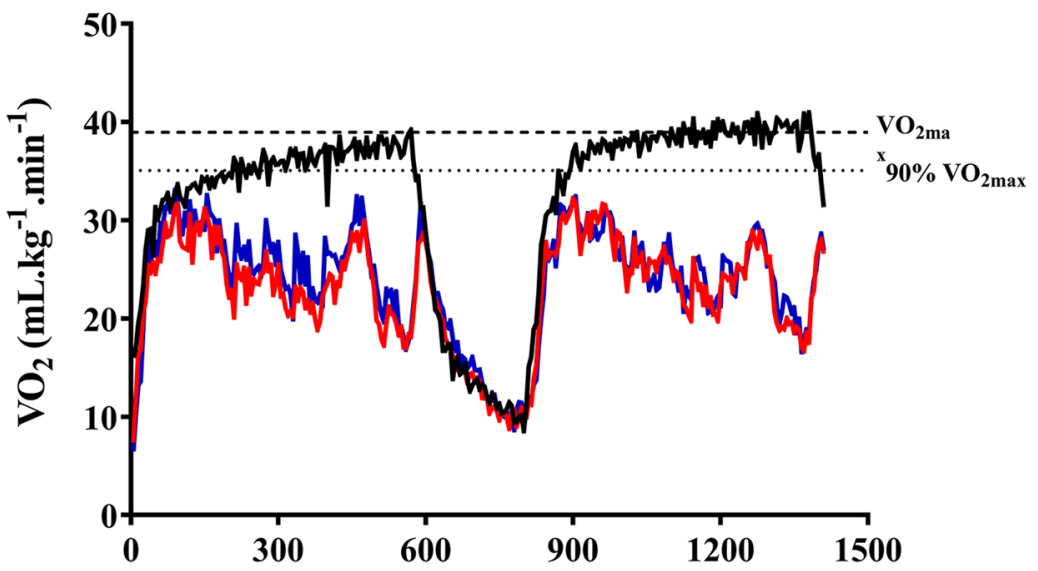

Time (s)
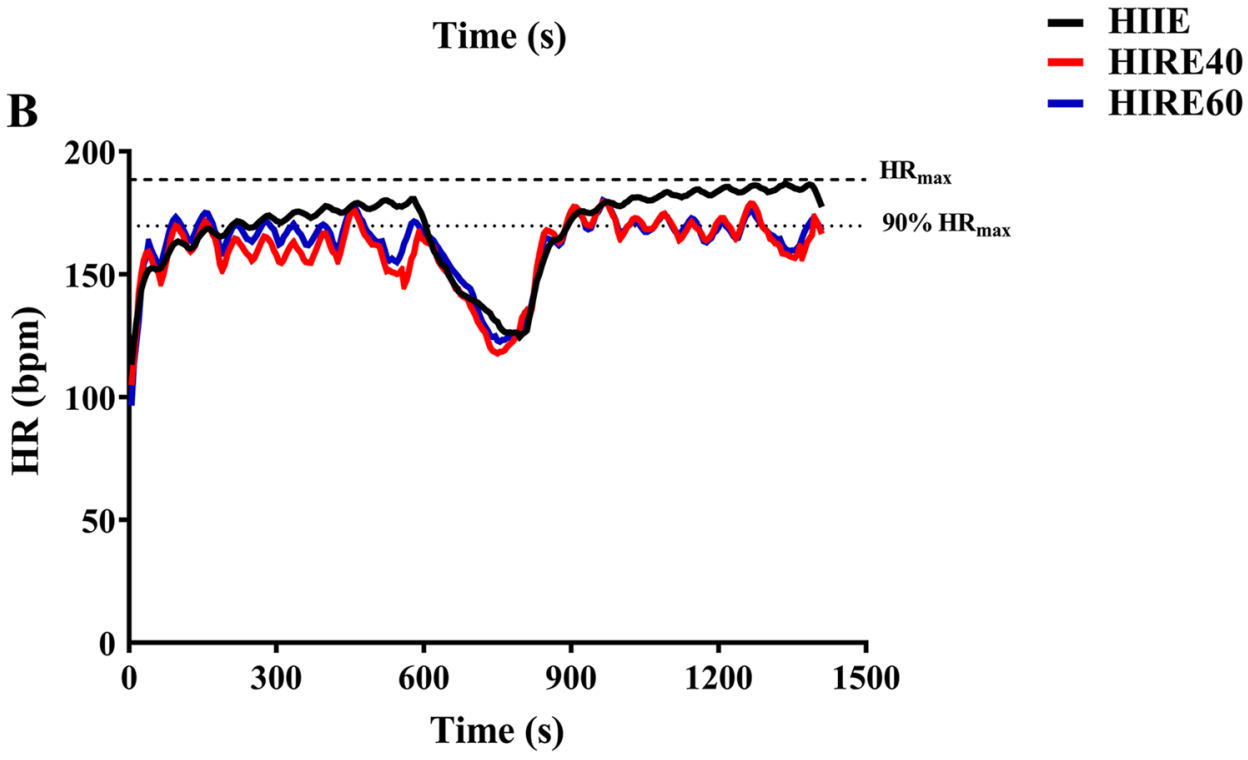

normality assumption. Skewed data were log-transformed and normal distribution was assessed again before selecting the appropriate parametric or non-parametric statistical tests. The following parameters were analyzed using oneway repeated measures analysis of variance (RM ANOVA): $\mathrm{VO}_{2 \text { max/peak }}$ (log-transformed), $\mathrm{VE}_{\text {max/peak }}, \mathrm{RER}_{\text {max/peak }}$, $\mathrm{VO}_{2 \text { mean }}$ [set 1 (log-transformed), set 2, average of sets 1 and 2, and rest], $\mathrm{VO}_{2 \text { peak }}$ (sets 1 and 2), $\mathrm{HR}_{\text {mean }}$ (set 1), $\mathrm{T} 90 \% \mathrm{HR}_{\max }$ (all time periods), blood lactate concentration (sets 1 and 2), RER (sets 1 and 2), RPE (set 1), EE (all measurements) and EPOC. When a significant main effect was observed, Bonferroni post hoc tests were used to evaluate the differences between the three exercise sessions. The following parameters were analyzed using Friedman's test: $\mathrm{HR}_{\text {max/peak }}, \mathrm{HR}_{\text {mean }}$ (set 2, average of sets 1 and 2, and rest), $\mathrm{HR}_{\text {peak }}$ (sets 1 and 2), $\mathrm{T} 90 \% \mathrm{VO}_{2 \max }$ (all time periods), and RPE (set 2). When a significant main effect was observed, Dunn's multiple comparisons tests were used to evaluate the differences between the three exercise sessions. Two-tailed paired $t$ tests were used to compare the number of repetitions and the load lifted between HIRE40 and HIRE60, and to compare the between-set changes in load lifted between HIRE40 and HIRE60. The $\alpha$-level of significance was set at $P<0.05$. Eta squared $\left(\eta^{2}\right)$ (for parametric tests) and Kendall W (for Friedman's tests) were determined to estimate the effect size. Effects sizes were classified as small $\left(\eta^{2}\right.$ : from 0.02 to 0.13 ; Kendall $\mathrm{W}$ : from 0.1 to 0.3$)$, moderate $\left(\eta^{2}\right.$ : from 0.13 to 0.26 ; Kendall $\mathrm{W}$ : from 0.3 to 0.5 ) and large $\left(\eta^{2}\right.$ : above 0.26 ; Kendall $\mathrm{W}$ : above 0.5$)$. A power analysis was performed from a pilot experiment (see the description above) using G*Power (version 3.1.9.4). The desired power was set to 0.8 and alpha was set at 0.05 . Power analyses performed on $\mathrm{T} 90 \mathrm{VO}_{2 \max }$ (average of sets 1 and 2) and $V \mathrm{O}_{2 \text { mean }}$ (average of sets 1 and 2) gave a sample size of 13 and 4 subjects, respectively. Fifteen subjects were included in the study, however, due to technical issues during the experiments (e.g., missing values of cardiorespiratory parameters and missing blood samples), the total number of participants 

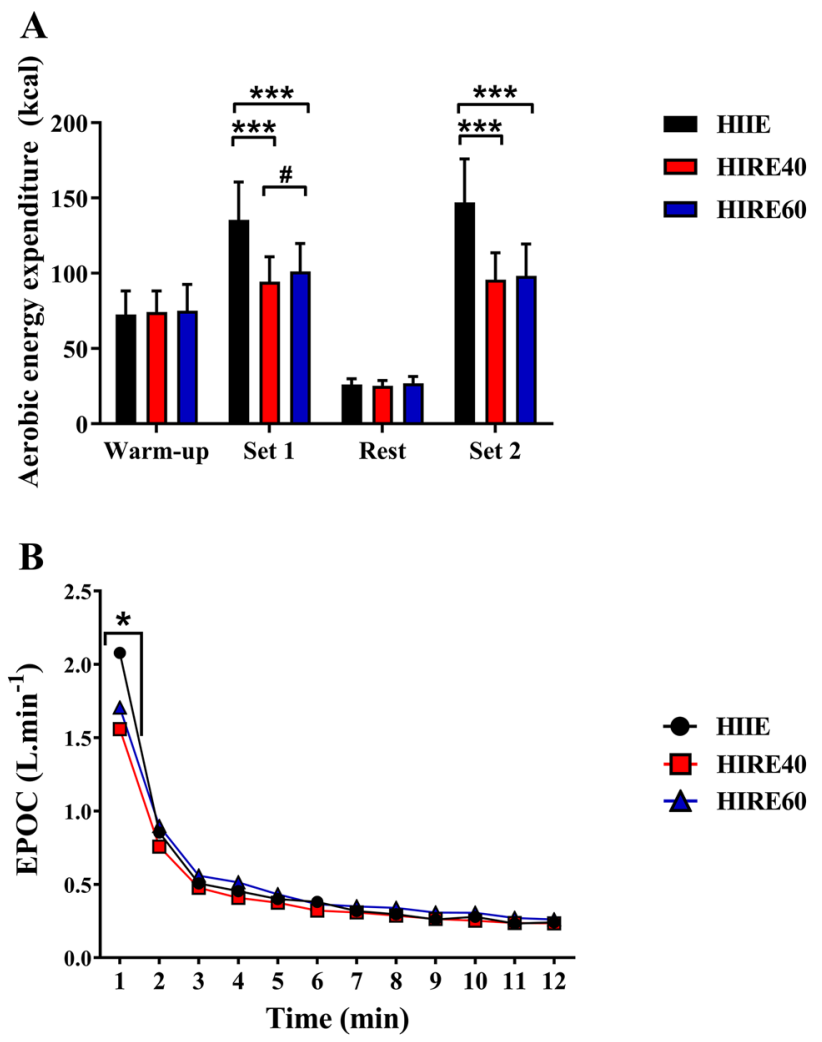

Fig. 2 Energy expenditure during the exercise session (A) and excess post-exercise oxygen consumption (EPOC) during the 12-min postexercise period (B) for the three interval exercise sessions. A Data are shown as mean $\pm \mathrm{SD}$. $N=14$ during the warm-up, $N=15$ during Set $1, N=14$ during rest, $N=12$ during Set 2 . $* * * P<0.001$, significantly different compared with HIIE. $\# P<0.05$, significant difference between HIRE40 and HIRE60. B Data are the mean values obtained from 11 participants. $* P<0.05$, significant difference between HIIE and HIRE40

included in the final analyses was between 11 and 15. The number of participants for each specific variable is presented in Table 5, and in the legend of Tables 2, 3 and 4 and Figs. 1 and 2.

\section{Results}

\section{Comparison of the cardiorespiratory parameters during the maximal incremental cycling test and the three interval exercises}

The highest values of $V \mathrm{O}_{2}, \mathrm{HR}, \mathrm{VE}$ and RER during the maximal incremental cycling test (maximal values) and the interval exercise sessions (peak values) are presented in Table 2. $V \mathrm{O}_{2 \max }$ (incremental test) was significantly higher than $V_{2 \text { peak }}$ observed during HIRE40 $(15 \%, P=0.008)$ and HIRE60 (13\%, $P=0.022$ ), while $V \mathrm{O}_{2 \max }$ was significantly lower than $V \mathrm{O}_{\text {2peak }}$ observed during HIIE $(-7 \%, P=0.013)$. $\mathrm{HR}_{\max }$ (incremental test) was significantly higher than
$\mathrm{HR}_{\text {peak }}$ observed during HIRE60 (5\%, $\left.P=0.039\right) . \mathrm{VE}_{\max }$ (incremental test) was significantly higher than $\mathrm{VE}_{\text {peak }}$ observed during HIRE40 and HIRE60 $(\sim 25 \%, P<0.01)$. $\mathrm{RER}_{\text {max }}$ (incremental test) was significantly higher than RER $_{\text {peak }}$ observed during HIIE $(14 \%, P<0.001)$, while $\mathrm{RER}_{\text {max }}$ was significantly lower than $\mathrm{RER}_{\text {peak }}$ observed during HIRE60 $(-8 \%, P=0.034)$.

\section{Cardiorespiratory response during the exercise sessions}

The changes in $V \mathrm{O}_{2}$ during the three exercise sessions are described in Fig. 1A. During HIIE, $V \mathrm{O}_{2}$ rapidly increased and was on average higher than $90 \% V \mathrm{O}_{2 \max }$ after approximately 4 and $1 \mathrm{~min}$ in set 1 and set 2 , respectively. During HIRE40 and HIRE60, $\mathrm{VO}_{2}$ rapidly increased over the first min of each set, and then highly fluctuated at a relatively moderate level. Overall, changes in $V \mathrm{O}_{2}$ were similar during HIRE40 and HIRE60. $V \mathrm{O}_{2}$ similarly decreased between the two sets for the three HIIE sessions.

$V \mathrm{O}_{2 \text { mean }}$ measured during HIRE40 and HIRE60 was substantially lower ( -25 to $30 \%)$ than during HIIE $(P<0.001$; Table 3). During set $1, V \mathrm{O}_{2 \text { mean }}$ was slightly lower $(-6 \%$, $P=0.032)$ in HIRE40 than in HIRE60. These differences were not found between HIRE40 and HIRE60 during set 2 and during the whole session (average of sets 1 and 2). $V \mathrm{O}_{\text {2mean }}$ was not different between the three sessions during the between-set recovery period (rest) (Table 3). $V \mathrm{O}_{2 \text { peak }}$ measured during HIRE40 and HIRE60 was lower $(\sim-15$ to $20 \%)$ than during HIIE $(P<0.001)$, with no significant differences found between HIRE40 and HIRE60 (Tables 2 and 3). $\mathrm{VO}_{2}$ was maintained above $90 \% \mathrm{VO}_{2 \max }$ over a prolonged period during both sets 1 and 2 for HIIE (Table 3 ). $\mathrm{T} 90 \% V \mathrm{O}_{2 \max }$ was very limited ( $\leq 1 \mathrm{~min}$ in total) during both HIRE40 and HIRE60, and was significantly lower than during HIIE $(P<0.001$ and $P=0.032$, respectively; Table 3$)$. $\mathrm{T} 90 \% V \mathrm{O}_{2 \max }$ was not significantly different between HIRE40 and HIRE60. During set 1, all subjects reached 90\% $V \mathrm{O}_{2 \max }$ in HIIE, while they were $6 / 11$ and $9 / 11$ to reach this level during HIRE40 and HIRE60, respectively. During set 2, all participants also reached $90 \% V \mathrm{O}_{2 \max }$ in HIIE, while they were $5 / 11$ and $7 / 11$ to reach $90 \% V \mathrm{O}_{2 \max }$ during HIRE40 and HIRE60, respectively.

The changes in HR during the three exercise sessions are described in Fig. 1B. During HIIE, HR rapidly increased and was on average higher than $90 \% \mathrm{HR}_{\max }$ after approximately 4 and 1 min $30 \mathrm{~s}$ in set 1 and set 2, respectively. During HIRE40 and HIRE60, HR rapidly increased over the first 3 min of each set, and then fluctuated during the remaining period of each set. Overall, the changes in HR were similar during HIRE40 and HIRE60. HR similarly decreased between sets 1 and 2 for the three sessions. 


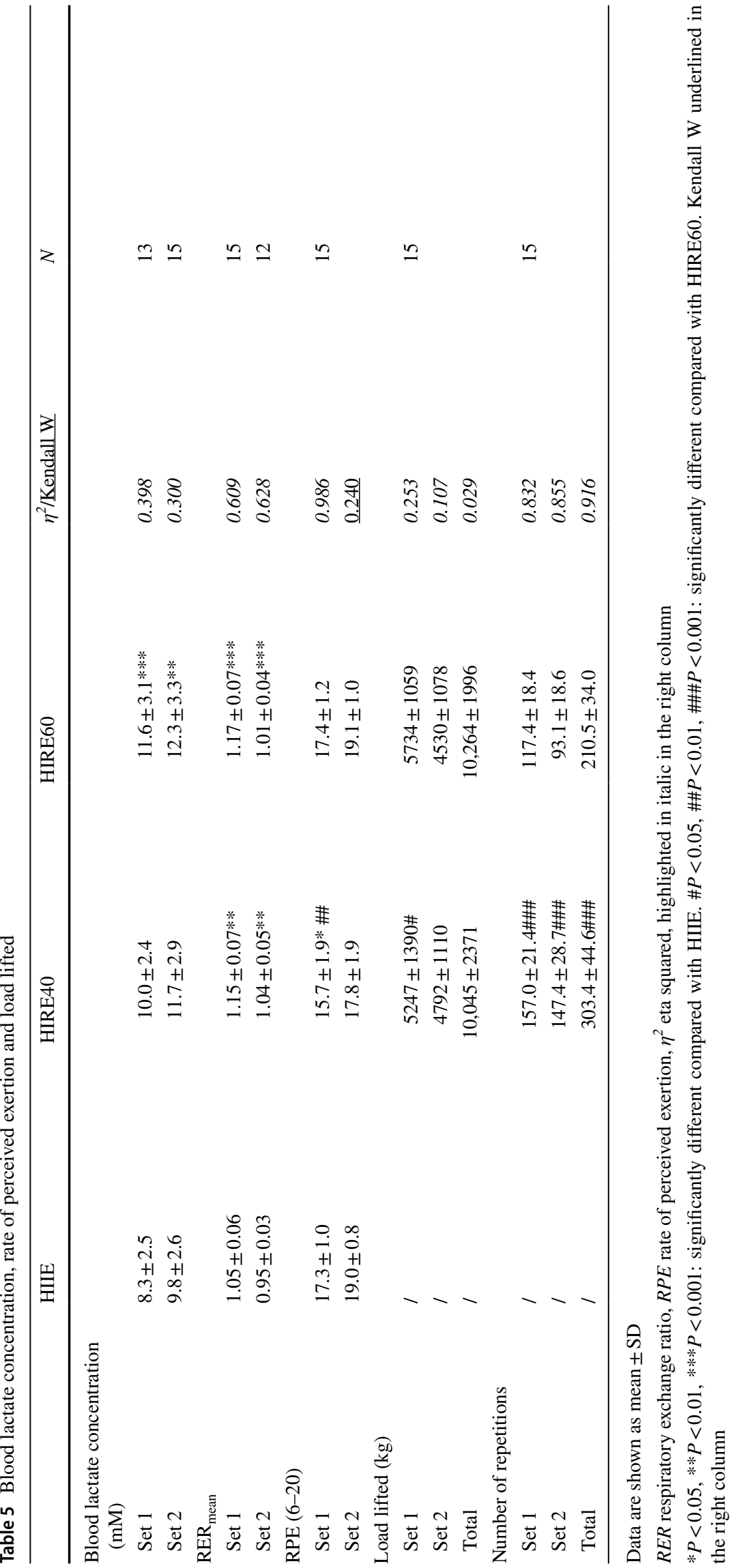


$\mathrm{HR}_{\text {mean }}$ measured during HIRE40 and HIRE60 was lower $(\sim-5 \%)$ than during HIIE $(P<0.05)$, with no significant differences found between HIRE40 and HIRE60 (Table 3). $\mathrm{HR}_{\text {mean }}$ was not different between the three sessions during the between-set recovery period (rest) (Table 3). During set $1, \mathrm{HR}_{\text {peak }}$ was not significantly different between HIIE, HIRE40 and HIRE60 (Table 3). During set 2, HR peak was slightly lower in HIRE60 compared with HIIE $(P=0.032)$, while no other significant differences were observed for this parameter. During sets 1 and 2, T90\% $\mathrm{HR}_{\max }$ was significantly lower for both HIRE40 and HIRE60 compared with HIIE $(P<0.05$; Table 3$)$. In total, T90\% $\mathrm{HR}_{\max }$ was $\sim 40 \%$ reduced during HIRE40 and HIRE60 compared with HIIE ( $P=0.003$ and $P<0.001$, respectively), while no significant differences were observed between HIRE40 and HIRE60. During both sets 1 and 2, all subjects reached $90 \% \mathrm{HR}_{\max }$ in HIIE, while they were $10 / 11$ to reach this level during HIRE40 and HIRE60.

\section{Energy expenditure}

Aerobic EE calculated during the three exercise sessions (including the warm-up, set 1, recovery between sets and set 2) is presented in Fig. 2A. Aerobic EE during the warmup and the in-between-set recovery period were similar for the three sessions. During sets 1 and 2, aerobic EE was significantly lower in HIRE40 and HIRE60 compared with HIIE (set 1: -25 to $30 \%, P<0.001 ; \eta^{2}=0.859$; set 2: 35\%, $\left.P<0.001, \eta^{2}=0.934\right)$. Aerobic EE was slightly lower in HIRE40 than HIRE60, but only during set $1(-7 \%$, $P=0.045)$.

Table 4 depicts the aerobic and anaerobic glycolytic EE during the exercise sessions (set $1+$ set 2). As shown in Fig. 2A, exercise aerobic EE was significantly lower in HIRE40 and HIRE60 compared with HIIE $(P<0.001)$ while no significant differences were found between the two HIRE sessions. Exercise anaerobic glycolytic EE was higher in HIRE60 than in HIIE $(P=0.007)$, whereas it was not different in HIRE40 compared with the two other interval exercises. Anaerobic glycolytic contribution to total exercise EE was higher in HIRE40 and HIRE60 compared with HIIE $(P<0.001)$, while the opposite result was found for the aerobic system $(P<0.001)$.

EPOC assessed during the 12-min post-exercise period is presented in Fig. 2B. EPOC rapidly decreased during the first min following the end of the exercises, and then remained relatively stable $(\sim 0.25 \mathrm{~L} / \mathrm{min})$ after $10 \mathrm{~min}$. EPOC was only significantly different between the exercise sessions at $1 \mathrm{~min}$ post-exercise (main effect: $P=0.018$; $\eta^{2}=0.410$ ), being significantly lower in HIRE40 compared with HIIE $(P=0.031)$. EE derived from EPOC (i.e., EE for aerobic ATP turnover and for the fast resynthesis of ATP/PC stores) was not significantly different between the exercise sessions (Table 4).

\section{Blood lactate concentration, RER, RPE and load lifted}

Blood lactate concentration was similar at baseline for the three exercise sessions (HIIE: $1.0 \pm 0.4 \mathrm{mmol} / \mathrm{L}$; HIRE40: $1.1 \pm 0.6 \mathrm{mmol} / \mathrm{L} ; \mathrm{HIRE} 60: 1.0 \pm 0.5 \mathrm{mmol} / \mathrm{L}$ ). After sets 1 and 2, blood lactate concentration was neither significantly different between HIIE and HIRE40, nor between HIRE40 and HIRE60, while it was significantly higher in HIRE60 than in HIIE $(P<0.001$; Table 5).

$\mathrm{RER}_{\text {peak }}$ was significantly higher in HIRE40 and HIRE60 compared with HIIE $(P<0.01$; Table 2$)$. $\mathrm{RER}_{\text {mean }}$ was significantly higher in HIRE40 and HIRE60 compared with HIIE during both set $1(P=0.003$ and $P<0.001$, respectively) and set $2(P=0.002$ and $P<0.001$, respectively), whereas no differences were observed between the two HIRE sessions (Table 5).

After set 1, RPE was significantly lower in HIRE40 compared to HIRE60 $(P=0.003)$ and HIIE $(P=0.015)$, while no differences were found between HIRE60 and HIIE (Table 5). No significant differences between the three exercise sessions were found for RPE after set 2.

The total number of repetitions was significantly lower during HIRE40 than during HIRE60 $(P<0.001$; Table 4$)$. The load lifted was significantly higher for HIRE60 than for HIRE40 during set $1(9 \%, P=0.047$; Table 5), while no differences were found between the two HIRE sessions during set 2 or in total. The decrease in load lifted during set 2 compared to set 1 was more pronounced for HIRE60 than for HIRE40 ( $-20.7 \pm 11.8 \%$ vs. $-6.9 \pm 13.0 \%, P=0.004$; $\left.\eta^{2}=0.454\right)$.

\section{Discussion}

Physiological responses to interval exercise are determined by numerous parameters, including the duration and intensity of the exercise and recovery periods, as well as the type of exercise (e.g., cycling, running, resistance exercise, etc.) (MacInnis and Gibala 2017). In this study, we examined the effect of the type of interval exercise (HIRE40 and HIRE60 vs. HIIE) on the cardiorespiratory response, and EE during and after exercise. In accordance with our hypothesis, we showed that the cardiorespiratory response to exercise was markedly reduced during HIRE40 and HIRE60 compared with HIIE, as demonstrated by lower $V \mathrm{O}_{2 \text { mean }}$, $V \mathrm{O}_{\text {2peak }}, \mathrm{T} 0 \% \mathrm{VO}_{2 \max }, \mathrm{HR}_{\text {mean }}, \mathrm{T} 90 \% \mathrm{HR}_{\text {max }}$ and $\mathrm{VE}_{\text {peak }}$. Aerobic exercise EE was also highly reduced in HIRE60 and HIRE40 compared with HIIE, while the anaerobic glycolytic contribution to total exercise EE was higher in HIRE than 
HIIE sessions. EPOC EE during the early recovery phase was similar in the three exercise sessions. In contrast to our hypothesis, the cardiorespiratory response, EE during exercise and EPOC were not substantially different between HIRE40 and HIRE60.

Most studies comparing acute physiological responses between different interval exercises focused on exercise modalities where protocols were matched for total work, or where objective intensity (i.e., power or speed) was controlled during interval exercises repeated over a fixed period or until exhaustion (Billat et al. 2000; Thevenet et al. 2007a, b, 2008; Rozenek et al. 2007; Smilios et al. 2018; Tucker et al. 2015). In the present study, the three exercise sessions had identical durations. However, exercise/recovery intensity was based on the power during HIIE, while the power could not be controlled and assessed during the two HIRE sessions due to their specific nature. Thus, working bouts of the HIRE sessions were executed at maximal sustainable work intensity and the intensity was assessed from the perceived effort (i.e., RPE) directly after the session. This method, which has been recently used by others (Rønnestad et al. 2020), constitutes a relevant approach applied to real world training. As shown in our pilot study (see in the method section), the present results confirmed that RPE was not different between HIIE and HIRE sessions directly after exercise, indicating that the perceived effort was comparable between the three interval exercises.

One of the main finding of this study is that $\mathrm{T} 90 \% V \mathrm{O}_{2 \max }$ was on average maintained during the major part of HIIE, a result in agreement with previous studies focusing on $30 \mathrm{~s} / 30 \mathrm{~s}$ HIIE (Thevenet et al. 2007a, 2008), while $\mathrm{T} 90 \% V \mathrm{O}_{2 \max }$ was on average $\leq 1$ min during HIRE40 and HIRE60. This result is consistent with the lower $V \mathrm{O}_{2 \text { mean }}$ observed during HIRE40 and HIRE60 compared with HIIE. Several factors may explain this difference in cardiorespiratory response. First, although $V \mathrm{O}_{2 \text { peak }}$ remained lower during HIRE40 and HIRE60 compared with HIIE, numerous participants reached $90 \% V_{2 \max }$ during the two HIRE sessions, but this high $V \mathrm{O}_{2}$ level could not be sustained. The highest $V \mathrm{O}_{2}$ were observed when exercise movements highly recruited lower limb muscles, while low $\mathrm{VO}_{2}$ levels were found when exercises mostly mobilized upper limbs and/ or trunk muscles (see Table 1; Fig. 1A). This observation is consistent with the idea that the level of $\mathrm{VO}_{2}$ measured during intense exercise is determined by the amount of muscle mass being recruited (Ballor et al. 1987; Orr et al. 2013). Second, differences in stroke volume and in heart rate (to a smaller extent; see Table 3) have probably impaired the increase in cardiac output during HIRE sessions compared with HIIE, thereby limiting oxygen consumption. Although not assessed in this study, the potential lower stroke volume during HIRE sessions may result from high-intensity muscle contractions (which can reduce preload and increased afterload) (Miles et al. 1987) and Valsalva maneuver associated with heavy lifting (which increases intrathoracic and intraabdominal pressures and impairs venous return) (Lentini et al. 1993). Other factors may be involved in the reduced oxygen consumption observed during HIRE compared with HIIE, including a greater peripheral vasoconstriction (and thus a lower arteriovenous $\mathrm{O}_{2}$ difference) due to a potential higher sympathetic activity during HIRE sessions, the recruitment of fiber types with different metabolic properties (which could affect muscle $\mathrm{O}_{2}$ extraction) (Cardinale et al. 2019), the nature of muscle contractions, as well as the feature of the 30-s recovery periods. Indeed, these recovery periods could not be identically performed during the HIRE sessions (transition to move to the next exercise station) and HIIE (cycling at 50\% $\mathrm{P}_{\max }$ ). Apparent reductions of $\mathrm{VO}_{2}$ and $\mathrm{HR}$ were found during the 30-s recovery periods of HIRE sessions (see Fig. 1), which certainly have affected the overall cardiorespiratory response. Finally, blood lactate concentration was significantly higher for HIRE60 than HIIE (values observed in HIRE40 being between those found in HIRE60 and HIIE), and RER mean $_{\text {and }}$ RER $_{\text {peak }}$ were higher in both HIRE sessions (being $>1.0$ for RER $_{\text {mean }}$ and $>1.35$ for $\mathrm{RER}_{\text {peak }}$ ) compared with HIIE. These findings indicate a larger stimulation of the anaerobic glycolytic system (as also shown in Table 4) during HIRE than HIIE sessions, which certainly contributes to explain the differences in cardiorespiratory response between HIRE and HIIE.

An intriguing result of this study is that $V \mathrm{O}_{2 \text { peak }}$ during HIIE was higher than $\mathrm{VO}_{2 \max }$. The question as to whether $V \mathrm{O}_{2 \max }$ measured during a maximal incremental test is a real maximal value has been previously raised (Schaun 2017). To our knowledge, this phenomenon has not been reported in studies with a similar protocol (i.e., HIIE including a fixed number of repetitions). However, other studies using HIIE performed until exhaustion have reported higher $\mathrm{VO}_{2}$ values during HIIE than during a maximal incremental test (Billat et al. 2000; Chang et al. 2020).

Aerobic EE have previously been assessed during interval exercise sessions consisting of various types of exercises (Schaun et al. 2018, 2017; Islam et al. 2018; Benito et al. 2016; Falcone et al. 2015), but to our knowledge, no studies have compared exercise EE between traditional HIIE and HIRE. Similarly to the cardiorespiratory response, aerobic EE was substantially lower during HIRE sessions compared with HIIE. In addition, the anaerobic glycolytic contribution to total exercise EE was larger during HIRE40 and HIRE60 than during HIIE, and this contribution is in the same range as that reported by others during HIRE (Benito et al. 2016). However, the higher activation of the anaerobic glycolytic system during HIRE sessions cannot offset the limited aerobic EE during exercise, and therefore total EE remains clearly reduced during HIRE sessions compared with HIIE session. Small differences for EPOC were only 
observed during the first minute of post-exercise recovery (see Fig. 2B) due to the larger level of $\mathrm{VO}_{2}$ at the end of the HIIE session. However, EPOC EE following exercise was not significantly different between the three exercise sessions. Since early EPOC provide indications of the fast resynthesis of ATP/PC stores (Scott 2006; Scott et al. 2011), our results suggest that the phosphagens metabolism was unlikely differentially affected between the three exercise sessions.

In contrast to our second hypothesis, no marked differences in cardiorespiratory response and energy expenditure were found between HIRE40 and HIRE60. Interestingly, $\mathrm{VO}_{2 \text { mean }}$ and aerobic EE were slightly lower in HIRE40 than HIRE60 during set 1, a result that corroborates with the differences observed for RPE and the load lifted during set 1 . The reduced load lifted during set 2 compared with set 1 was more important in HIRE60 than HIRE40, but the cumulated load lifted was similar during both sessions. Therefore, HIRE60 appears to induce more fatigue and is more demanding than HIRE40 during set 1 , while the perceived effort and total work achieved at the end of both sessions are similar. This latter result most likely explains the absence of differences of cardiorespiratory response and energy expenditure between the two HIRE sessions. The contribution of anaerobic glycolysis to exercise EE, blood lactate concentration during exercise, and EPOC were not different between HIRE40 and HIRE60, which further indicate that overall, HIRE40 and HIRE60 induced a similar physiological response, at least for the variables analyzed in this study.

\section{Study limitations}

A few limitations should be highlighted from this study. First, we intentionally selected exercises soliciting various muscle groups to limit the accumulation of excessive fatigue that characterized HIRE. However, the choice of exercises was limited since the movements were executed with a mobile gas analyzer and using free weights. Although other exercises may have been more advantageous to reach and maintain high levels of $V \mathrm{O}_{2}$, it is unlikely that those exercises (i.e., movements mostly recruiting lower limb muscles) could have been executed at maximal intensity during 10 bouts of $30 \mathrm{~s}$ by recreationally active subjects. Another limitation is the loss of data of cardiorespiratory parameters during and after exercise, which was due to technical issues with our mobile gas analyzer. Our sample size (11 to 15 subjects included in the final analyses) was close to or higher than the estimated sample size calculated from our power analysis (see statistical analysis), and the effect sizes observed in this study were large for most variables. This indicates that our statistical analysis was certainly not underpowered. Furthermore, the anaerobic glycolytic EE was estimated using the $\mathrm{O}_{2}$ equivalent of blood lactate accumulation, a method widely used (Benito et al. 2016; di Prampero and Ferretti 1999; Bertuzzi et al. 2010). Although informative, it may not precisely estimate the contribution of the anaerobic glycolytic system in our study due to (1) our exercise protocol (e.g., alternance of intervals, intensity not controlled during HIRE sessions), (2) our experimental design ([La-] should have been measured at several time-points during exercise and rest) and 3) other limitations (e.g., the metabolic demand of active muscles may not be accurately estimated by analyzing whole-body physiological responses) (Bertuzzi et al. 2010). Finally, although ATP/PC component constitutes a part of EPOC (Scott 2006), some limitations regarding the use of EPOC has been reported (Green and Dawson 1993). Future works should develop better methods to estimate the anaerobic contribution during interval exercises.

\section{Practical implications and perspectives}

Our results clearly demonstrate that different types of interval exercise can induce various physiological responses in recreationally active subjects. Therefore, a specific interval exercise session should be chosen in accordance with the training purpose. If the main objective is to perform a short and intense session that highly solicits the cardiorespiratory system and markedly increases EE, HIIE using large muscle groups (e.g., cycling, running, rowing, etc.) should be advised. HIRE should be rather encouraged for strength and conditioning training, allowing to strengthen different muscle groups while moderately activating the cardiorespiratory system and increasing EE. However, some recommendations could be provided to optimize $\mathrm{T} 90 \% \mathrm{VO}_{2 \max }$ during HIRE. First, selected exercise movements should recruit major muscle groups and/or engage several muscle groups together (e.g., lower limb with upper limb/trunk muscles), while avoiding the execution of successive exercises that mobilize similar muscle groups to limit the accumulation of excessive fatigue. Second, exercise bouts should be performed at maximal intensity (i.e., execution of the highest number of repetitions for each movement) while maintaining a proper technique to avoid any risks of injury. Since the intensity was not sustainable during the set 2 of HIRE60 (see Table 4), it may be more appropriate to perform HIRE at $40 \% 1-\mathrm{RM}$, at least in recreationally active subjects. Finally, it may also be relevant to shorten the duration of the sets to limit the accumulation of fatigue (e.g., $6 \mathrm{~min}$ instead of $10 \mathrm{~min}$ ) while increasing the number of sets 
per session. This option could be helpful to progressively increase the working time over a training intervention. Although our results indicate that HIRE does not induce an optimal stimulus on the cardiorespiratory system for improving $V \mathrm{O}_{2 \max }$, recent studies have demonstrated that high-intensity functional training enables to develop it (Munoz-Martinez et al. 2017; McRae et al. 2012). Future studies should compare the improvement of $V \mathrm{O}_{2 \max }$ after a training intervention including HIIE or HIRE.

\section{Conclusion}

Our study demonstrates that HIRE is not as effective as HIIE for increasing the cardiorespiratory response and EE during exercise in recreationally active subjects. However, EPOC EE during the early recovery phase is similar in HIRE and HIIE sessions. Our results also show that the cardiorespiratory response and EE during exercise, and EPOC are not substantially different between HIRE performed with low or moderate load.

Acknowledgements The authors thank the participants of the study for their time and engagement, and the staff of Actic Örebro Slottsgatan and Örebro Kraftsportklubb for access to their facilities. The authors thank Ms. Kristina Sutela and Mr. Igor Sanna for careful reading of the manuscript, and Mr. Diego Montiel-Rojas for helpful discussions related to statistical analyses.

Author contributions LJ, SLP and TC conceived and designed research. LJ and SLP performed experiments. SLP performed the pilot experiment. LJ, SLP and TC analyzed data. LJ, SLP and TC interpreted the results, prepared the figures, and drafted the manuscript. LJ and TC edited and revised the manuscript. LJ, SLP and TC approved the final version of the manuscript.

Funding Open access funding provided by Örebro University.

Availability of data and materials On request.

\section{Declarations}

Conflict of interest The authors declare no conflicts of interest.

Ethics approval This study was performed in accordance with the declaration of Helsinki and was approved by the regional ethics committee of Uppsala (DNR 2018/321)

Consent to participate The participants were fully informed of the study procedures and a written informed consent was obtained from all participants.

Consent for publication Not applicable.

Open Access This article is licensed under a Creative Commons Attribution 4.0 International License, which permits use, sharing, adaptation, distribution and reproduction in any medium or format, as long as you give appropriate credit to the original author(s) and the source, provide a link to the Creative Commons licence, and indicate if changes were made. The images or other third party material in this article are included in the article's Creative Commons licence, unless indicated otherwise in a credit line to the material. If material is not included in the article's Creative Commons licence and your intended use is not permitted by statutory regulation or exceeds the permitted use, you will need to obtain permission directly from the copyright holder. To view a copy of this licence, visit http://creativecommons.org/licenses/by/4.0/.

\section{References}

Ballor DL, Becque MD, Katch VL (1987) Metabolic responses during hydraulic resistance exercise. Med Sci Sports Exerc 19(4):363-367

Benito PJ, Alvarez-Sanchez M, Diaz V, Morencos E, Peinado AB, Cupeiro R, Maffulli N (2016) Cardiovascular Fitness and Energy Expenditure Response during a Combined Aerobic and Circuit Weight Training Protocol. PLoS ONE. https://doi.org/10.1371/ journal.pone.0164349

Bertuzzi RC, Franchini E, Ugrinowitsch C, Kokubun E, Lima-Silva AE, Pires FO, Nakamura FY, Kiss MA (2010) Predicting MAOD using only a supramaximal exhaustive test. Int J Sports Med 31(7):477-481. https://doi.org/10.1055/s-0030-1253375

Billat VL, Slawinski J, Bocquet V, Demarle A, Lafitte L, Chassaing P, Koralsztein JP (2000) Intermittent runs at the velocity associated with maximal oxygen uptake enables subjects to remain at maximal oxygen uptake for a longer time than intense but submaximal runs. Eur J Appl Physiol 81(3):188-196. https://doi.org/10.1007/ s004210050029

Buchheit M, Laursen PB (2013) High-intensity interval training, solutions to the programming puzzle: Part I: cardiopulmonary emphasis. Sports Med 43(5):313-338. https://doi.org/10.1007/ s40279-013-0029-x

Cardinale DA, Larsen FJ, Jensen-Urstad M, Rullman E, Søndergaard H, Morales-Alamo D, Ekblom B, Calbet JAL, Boushel R (2019) Muscle mass and inspired oxygen influence oxygen extraction at maximal exercise: role of mitochondrial oxygen affinity. Acta Physiol (oxf). https://doi.org/10.1111/apha.13110

Chang SC, Adami A, Lin HC, Lin YC, Chen CPC, Fu TC, Hsu CC, Huang SC (2020) Relationship between maximal incremental and high-intensity interval exercise performance in elite athletes. PLoS ONE 15(5):e0226313. https://doi.org/10.1371/journal.pone. 0226313

Claudino JG, Gabbett TJ, Bourgeois F, Souza HS, Miranda RC, Mezêncio B, Soncin R, Cardoso Filho CA, Bottaro M, Hernandez AJ, Amadio AC, Serrão JC (2018) CrossFit overview: systematic review and meta-analysis. Sports Med Open 4(1):11. https://doi. org/10.1186/s40798-018-0124-5

di Prampero PE, Ferretti G (1999) The energetics of anaerobic muscle metabolism: a reappraisal of older and recent concepts. Respir Physiol 118(2-3):103-115. https://doi.org/10.1016/ s0034-5687(99)00083-3

Falcone PH, Tai CY, Carson LR, Joy JM, Mosman MM, McCann TR, Crona KP, Kim MP, Moon JR (2015) Caloric expenditure of aerobic, resistance, or combined high-intensity interval training using a hydraulic resistance system in healthy men. J Strength Cond Res 29(3):779-785. https://doi.org/10.1519/jsc.00000 00000000661

Feito Y, Heinrich KM, Butcher SJ, Poston WSC (2018) High-intensity functional training (HIFT): definition and research implications for improved fitness. Sports (basel). https://doi.org/10.3390/sport s6030076 
Gaesser GA, Brooks GA (1984) Metabolic bases of excess postexercise oxygen consumption: a review. Med Sci Sports Exerc 16(1):29-43

Gibala MJ (2020) Physiological basis of interval training for performance enhancement. Exp Physiol. https://doi.org/10.1113/ep088 190

Green S, Dawson B (1993) Measurement of anaerobic capacities in humans. Definitions, limitations and unsolved problems. Sports Med 15(5):312-327. https://doi.org/10.2165/00007256-19931 5050-00003

Islam H, Townsend LK, Hazell TJ (2018) Excess postexercise oxygen consumption and fat utilization following submaximal continuous and supramaximal interval running. Res Q Exerc Sport 89(4):450-456. https://doi.org/10.1080/02701367.2018.1513633

Jung WS, Hwang H, Kim J, Park HY, Lim K (2019) Effect of interval exercise versus continuous exercise on excess post-exercise oxygen consumption during energy-homogenized exercise on a cycle ergometer. J Exerc Nutrition Biochem 23(2):45-50. https://doi. org/10.20463/jenb.2019.0016

Keating SE, Johnson NA, Mielke GI, Coombes JS (2017) A systematic review and meta-analysis of interval training versus moderate-intensity continuous training on body adiposity. Obes Rev 18(8):943-964. https://doi.org/10.1111/obr.12536

Lentini AC, McKelvie RS, McCartney N, Tomlinson CW, MacDougall JD (1993) Left ventricular response in healthy young men during heavy-intensity weight-lifting exercise. J Appl Physiol 75(6):2703-2710. https://doi.org/10.1152/jappl.1993.75.6.2703

MacInnis MJ, Gibala MJ (2017) Physiological adaptations to interval training and the role of exercise intensity. J Physiol 595(9):29152930. https://doi.org/10.1113/jp273196

Mayhew JL, Prinster JL, Ware JS, Zimmer DL, Arabas JR, Bemben MG (1995) Muscular endurance repetitions to predict bench press strength in men of different training levels. J Sports Med Phys Fitness 35(2):108-113

McRae G, Payne A, Zelt JG, Scribbans TD, Jung ME, Little JP, Gurd BJ (2012) Extremely low volume, whole-body aerobic-resistance training improves aerobic fitness and muscular endurance in females. Appl Physiol Nutr Metab 37(6):1124-1131. https://doi. org/10.1139/h2012-093

Midgley AW, McNaughton LR, Wilkinson M (2006) Is there an optimal training intensity for enhancing the maximal oxygen uptake of distance runners?: empirical research findings, current opinions, physiological rationale and practical recommendations. Sports Med 36(2):117-132

Miles DS, Owens JJ, Golden JC, Gotshall RW (1987) Central and peripheral hemodynamics during maximal leg extension exercise. Eur J Appl Physiol Occup Physiol 56(1):12-17. https://doi.org/ 10.1007/bf00696369

Millet GP, Candau R, Fattori P, Bignet F, Varray A (2003a) VO2 responses to different intermittent runs at velocity associated with VO2max. Can J Appl Physiol 28(3):410-423. https://doi.org/10. 1139/h03-030

Millet GP, Libicz S, Borrani F, Fattori P, Bignet F, Candau R (2003b) Effects of increased intensity of intermittent training in runners with differing VO2 kinetics. Eur J Appl Physiol 90(1-2):50-57. https://doi.org/10.1007/s00421-003-0844-0

Munoz-Martinez FA, Rubio-Arias JA, Ramos-Campo DJ, Alcaraz PE (2017) Effectiveness of resistance circuit-based training for maximum oxygen uptake and upper-body one-repetition maximum improvements: a systematic review and meta-analysis. Sports Med 47(12):2553-2568. https://doi.org/10.1007/s40279-017-0773-4

Myers TR, Schneider MG, Schmale MS, Hazell TJ (2015) Whole-body aerobic resistance training circuit improves aerobic fitness and muscle strength in sedentary young females. J Strength Cond Res 29(6):1592-1600. https://doi.org/10.1519/jsc.0000000000000790
Orr JL, Williamson P, Anderson W, Ross R, McCafferty S, Fettes P (2013) Cardiopulmonary exercise testing: arm crank vs cycle ergometry. Anaesthesia 68(5):497-501. https://doi.org/10.1111/ anae. 12195

Pinckard K, Baskin KK, Stanford KI (2019) Effects of exercise to improve cardiovascular health. Front Cardiovasc Med 6:69. https://doi.org/10.3389/fcvm.2019.00069

Rønnestad BR, Hansen J (2016) Optimizing interval training at power output associated with peak oxygen uptake in well-trained cyclists. J Strength Cond Res 30(4):999-1006. https://doi.org/10. 1519/JSC.0b013e3182a73e8a

Rønnestad BR, Hansen J, Nygaard H, Lundby C (2020) Superior performance improvements in elite cyclists following short-interval vs effort-matched long-interval training. Scand J Med Sci Sports 30(5):849-857. https://doi.org/10.1111/sms.13627

Rosdahl H, Gullstrand L, Salier-Eriksson J, Johansson P, Schantz P (2010) Evaluation of the Oxycon Mobile metabolic system against the Douglas bag method. Eur J Appl Physiol 109(2):159-171. https://doi.org/10.1007/s00421-009-1326-9

Rozenek R, Funato K, Kubo J, Hoshikawa M, Matsuo A (2007) Physiological responses to interval training sessions at velocities associated with VO2max. J Strength Cond Res 21(1):188-192. https:// doi.org/10.1519/r-19325.1

Schaun GZ (2017) The maximal oxygen uptake verification phase: a light at the end of the tunnel? Sports Med Open 3(1):44. https:// doi.org/10.1186/s40798-017-0112-1

Schaun GZ, Alberton CL, Ribeiro DO, Pinto SS (2017) Acute effects of high-intensity interval training and moderate-intensity continuous training sessions on cardiorespiratory parameters in healthy young men. Eur J Appl Physiol 117(7):1437-1444. https://doi.org/10. 1007/s00421-017-3636-7

Schaun GZ, Pinto SS, Praia ABC, Alberton CL (2018) Energy expenditure and EPOC between water-based high-intensity interval training and moderate-intensity continuous training sessions in healthy women. J Sports Sci 36(18):2053-2060. https://doi.org/10.1080/ 02640414.2018 .1435967

Scott CB (2006) Contribution of blood lactate to the energy expenditure of weight training. J Strength Cond Res 20(2):404-411. https://doi.org/10.1519/r-17495.1

Scott CB, Leighton BH, Ahearn KJ, McManus JJ (2011) Aerobic, anaerobic, and excess postexercise oxygen consumption energy expenditure of muscular endurance and strength: 1-set of bench press to muscular fatigue. J Strength Cond Res 25(4):903-908. https://doi.org/10.1519/JSC.0b013e3181c6a128

Shimano T, Kraemer WJ, Spiering BA, Volek JS, Hatfield DL, Silvestre R, Vingren JL, Fragala MS, Maresh CM, Fleck SJ, Newton RU, Spreuwenberg LP, Häkkinen K (2006) Relationship between the number of repetitions and selected percentages of one repetition maximum in free weight exercises in trained and untrained men. J Strength Cond Res 20(4):819-823. https://doi.org/10. $1519 / \mathrm{r}-18195.1$

Skelly LE, Andrews PC, Gillen JB, Martin BJ, Percival ME, Gibala MJ (2014) High-intensity interval exercise induces 24-h energy expenditure similar to traditional endurance exercise despite reduced time commitment. Appl Physiol Nutr Metab 39(7):845848. https://doi.org/10.1139/apnm-2013-0562

Skrypnik D, Bogdański P, Mądry E, Karolkiewicz J, Ratajczak M, Kryściak J, Pupek-Musialik D, Walkowiak J (2015) Effects of endurance and endurance strength training on body composition and physical capacity in women with abdominal obesity. Obes Facts 8(3):175-187. https://doi.org/10.1159/000431002

Smilios I, Myrkos A, Zafeiridis A, Toubekis A, Spassis A, Tokmakidis SP (2018) The effects of recovery duration during highintensity interval exercise on time spent at high rates of oxygen 
consumption, oxygen kinetics, and blood lactate. J Strength Cond Res 32(8):2183-2189. https://doi.org/10.1519/jsc.0000000000 001904

Thevenet D, Tardieu-Berger M, Berthoin S, Prioux J (2007a) Influence of recovery mode (passive vs active) on time spent at maximal oxygen uptake during an intermittent session in young and endurance-trained athletes. Eur J Appl Physiol 99(2):133-142. https://doi.org/10.1007/s00421-006-0327-1

Thevenet D, Tardieu M, Zouhal H, Jacob C, Abderrahman BA, Prioux J (2007b) Influence of exercise intensity on time spent at high percentage of maximal oxygen uptake during an intermittent session in young endurance-trained athletes. Eur J Appl Physiol 102(1):19-26. https://doi.org/10.1007/s00421-007-0540-6

Thevenet D, Leclair E, Tardieu-Berger M, Berthoin S, Regueme S, Prioux J (2008) Influence of recovery intensity on time spent at maximal oxygen uptake during an intermittent session in young, endurance-trained athletes. J Sports Sci 26(12):1313-1321. https://doi.org/10.1080/02640410802072697

Tiggemann CL, Korzenowski AL, Brentano MA, Tartaruga MP, Alberton CL, Kruel LF (2010) Perceived exertion in different strength exercise loads in sedentary, active, and trained adults. J Strength Cond Res 24(8):2032-2041. https://doi.org/10.1519/JSC.0b013 e3181d32e29

Tucker WJ, Sawyer BJ, Jarrett CL, Bhammar DM, Gaesser GA (2015) Physiological responses to high-intensity interval exercise differing in interval duration. J Strength Cond Res 29(12):3326-3335. https://doi.org/10.1519/jsc.0000000000001000

Wen D, Utesch T, Wu J, Robertson S, Liu J, Hu G, Chen H (2019) Effects of different protocols of high intensity interval training for $\mathrm{VO}(2)$ max improvements in adults: a meta-analysis of randomised controlled trials. J Sci Med Sport 22(8):941-947. https://doi.org/ 10.1016/j.jsams.2019.01.013

Zinoubi B, Zbidi S, Vandewalle H, Chamari K, Driss T (2018) Relationships between rating of perceived exertion, heart rate and blood lactate during continuous and alternated-intensity cycling exercises. Biol Sport 35(1):29-37. https://doi.org/10.5114/biols port.2018.70749

Publisher's Note Springer Nature remains neutral with regard to jurisdictional claims in published maps and institutional affiliations. 